\title{
Holographic Superconductivity in the Presence of Dark Matter: Basic Issues
}

\author{
M. RogatKo* AND K.I. WYSOKIŃSKI \\ Institute of Physics, Maria Curie-Skłodowska University, Pl. Marii Curie-Skłodowskiej 1, 20-031 Lublin, Poland
}

\begin{abstract}
The holographic approach to study strongly coupled superconductors in the presence of dark matter is reviewed. We discuss the influence of dark matter on the superconducting transition temperature of both $s$-wave and $p$ wave holographic superconductors. The upper critical field, coherence length, penetration depth of holographic superconductors as well as the metal-insulator transitions have also been analysed. Issues related to the validity of anti-de Sitter/conformal field theory correspondence for the description of superconductors studied in the laboratory and possible experiments directed towards the detection of dark matter are discussed. In doing so we shall compare our assumptions and assertions with those generally accepted in the elementary particle experiments aimed at the detection of dark matter particles.
\end{abstract}

DOI: 10.12693/APhysPolA.130.558

PACS/topics: $11.25 . \mathrm{Tq}, 04.50 .-\mathrm{h}, 98.80 . \mathrm{Cq}$

\section{Introduction}

The superconductivity is a macroscopic quantum phenomenon [1] appearing in many condensed matter systems and characterized by the expulsion of the magnetic field and zero resistance state at sufficiently low temperature. The superconducting rings show quantization of magnetic flux. This is also true for vortices in the type two superconductors. A number of other quantum effects appear if the superconductor is in contact with normal metal (the Andreev reflection) or other superconductors (the Josephson effect). All these properties and phenomena are at the heart of numerous applications of superconductors.

Zero resistivity state allows the construction and operation of powerful superconducting electromagnets for important scientific and medical applications. The superconducting quantum interference devices (SQUIDs) are the most sensitive magnetometers. SQUIDs allow for the precise measurements of the flux quantum $\phi=h / 2 e$, where $e$ is the electron charge and $h$ - the Planck constant. These devices have been proposed and used in the search for the magnetic monopole, the elementary particle postulated [2] by Dirac in 1931. The search [3] for this elusive particle continues [4], with the hope that it might address an important question of composition of dark matter [5].

According to standard theory of gravity based on Newtonian dynamics the explanation of many astrophysical observations require the existence of large amount of matter that cannot be seen with telescopes, and is thus termed dark matter. The Planck satellite mission reveals that dark matter constitutes $26.8 \%$ of the total mass of the Universe, while ordinary matter makes only $4.9 \%$ of it. The rest [6] is the mysterious dark energy, $68.3 \%$.

\footnotetext{
* corresponding author; e-mail: rogat@kft.umcs.lublin.pl
}

The nature of the dark matter is still unknown. Besides magnetic monopoles other particles have been proposed as possible candidates. Many experiments [7] aimed at the direct detection of the postulated dark matter particles have been proposed. Some of them $[8,9]$ rely on the use of superconductors and study of subtle quantum (interference) effects. We shall not discuss various proposals for the dark matter particles [10] here. Instead we model the dark matter classically by the additional field with properties similar to the Maxwell field and study the influence of dark matter on the properties of holographic superconductors. The main goal of the present work is to discuss how the presence of dark matter may change the properties of holographic superconductors. Assuming that the holographic analogy teaches us about the strong coupling aspects of superconductors studied in the laboratory we discuss possible experiments directed towards the detection of this elusive but dominant part of matter in the Universe.

The rest of the paper is organized as follows. We discuss the main ideas of the general holographic approach to study strongly coupled models in Sect. 2, where we also briefly state the main effects of the dark sector on the properties of superconductors. In Sect. 3 we shall discuss general questions of matter composition and the geometry of the Universe, chances of direct detection of dark matter field in the laboratory by long time observations of superconductors. We also offer some remarks on the possibility of superconducting condensation of dark matter. We end up with conclusions.

\section{The effect of dark matter on superconductors within AdS/CFT correspondence}

The gauge/gravity duality is also known as holographic duality or anti-de Sitter/conformal field theory (AdS/CFT) correspondence [11]. It is the conjectured equivalence between the field theory in the Minkowski 
space-time forming the boundary of the Einstein - antide Sitter (AdS) and the gravity theory in the bulk of the AdS space-time, see Fig. 1. There exists a kind of a "dictionary" which relates the results of calculations in one theory to those obtained or expected in the other. In other words, every property in one theory has a counterpart in the other theory. In order to study temperature dependent phenomena one assumes the existence of black hole in the gravity theory. The AdS/CFT correspondence is typically viewed as a prescription how to calculate the expectation values of the operators in the strongly coupled field theory by means of the gravity theory. The latter is typically easy endeavor as the gravity action is dominated by classic field configurations. It is believed that the correspondence provides the strong couplingweak coupling duality [12]. In technical terms [13] the correspondence is related to the fact that the partition function of the field theory $Z[A(\boldsymbol{x}, t)]$, where $A(\boldsymbol{x}, t)$ is the source field that couples to the currents $\boldsymbol{j}(\boldsymbol{x}, t)$, equals to that of the gravity dual $Z[A(\boldsymbol{x}, r, t)]$ with the boundary condition $\lim _{r \rightarrow \infty} A(\boldsymbol{x}, r, t)=A(\boldsymbol{x}, t)$. As mentioned the latter partition function is prevailed by the classical field configurations of gravitational field and thus is given by the exponential of the classical action of the aforementioned configurations.

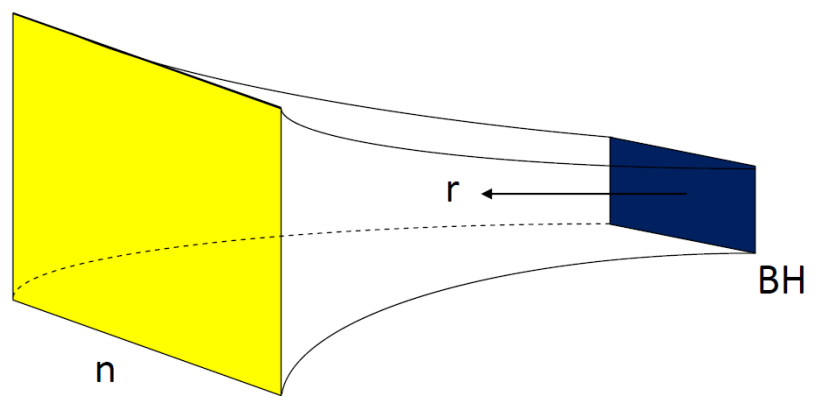

Fig. 1. In the holographic approach the shadowed plane on the right represents the black hole (BH). The extra "radial" dimension is denoted by $r$. The light shadowed plane on the left represents the boundary $r \rightarrow \infty$ of the AdS $n$-dimensional space-time, i.e. for $n=5$ the Minkowski space $(t, x, y, z)$ of the field theory. The region of finite $r(0<r<\infty)$ is denoted as gravitational bulk. All processes in the bulk represent long distance or low energy processes (IR limit), while those close to boundary correspond to short distance or high energies (UV limit). Thus distance $r$ correspond to the renormalisation group parameter deciding about the coupling strength.

The first successful description of strongly coupled superconductors by gauge/gravity duality [14] relied on the planar Schwarzschild-anti-de Sitter black hole and Maxwell electrodynamics. The scalar complex field $\Psi(r)$ is coupled to the Maxwell field represented by the time component of the four-vector $A_{t}=\Phi(r)$. It has to be stressed that properties of the dual field theory can be read off from the asymptotic behavior $r \rightarrow \infty$ of the field equations. In particular, the scalar field behaves close to the boundary $r \rightarrow \infty$ as $\Psi(r)=\left\langle O_{1}\right\rangle / r+\left\langle O_{2}\right\rangle / r^{2}+\ldots$ where according to the general prescription $\left\langle O_{1}\right\rangle$ and $\left\langle\mathrm{O}_{2}\right\rangle$ play a role of the condensate density in the dual theory and can be compared with superconducting order parameter. Both $\left\langle O_{1}\right\rangle$ and $\left\langle O_{2}\right\rangle$ take on the non-zero value at temperatures (provided by the black hole) lower than the characteristic critical temperature $T_{c}$. The temperature dependence of $\left\langle\mathrm{O}_{2}\right\rangle$ close to $T_{c}$ shows the behavior characteristic for the superconducting order parameter $\Delta$ with the mean-field type dependence $\propto\left(1-T / T_{c}\right)^{1 / 2}$ close to $T_{c}$.

\subsection{Strong coupling effects in holographic superconductors}

The calculated [14] ratio $2 \sqrt{\left\langle O_{2}(T=0)\right\rangle} / T_{c} \approx 8$ is characteristic for strongly coupled superconductors. The other strong coupling effects include the upward curvature seen in the temperature dependence of the upper critical field calculated via AdS/CFT correspondence $[15-19]$. It leads to the value of zero temperature magnetic field higher than the estimate based on the equation [20]: $B_{c}(0) \approx 0.69 T_{c}\left(\frac{\mathrm{d} B_{c}(T)}{\mathrm{d} T}\right)_{T_{c}}$ relating upper critical field at $T=0$ to its derivative close to $T_{c}$. The upward curvature, which is observed in many high temperature superconductors in the standard approach [20] requires inter alia strong potential or spin-orbit scattering, while it seems to appear quite naturally in the strong coupling.

\subsection{Generalizations}

Since the first attempt to apply the gravity to construct holographic superconductors [14], there appeared a number of papers generalizing the approach in various directions. Among them one has to mention the complex gravity backgrounds including higher order curvatures [21], non-linear Born-Infeld electrodynamics [22], etc. Important results have been obtained by changing the chemical potential $\mu$, which in the gauge/gravity theory is given by the asymptotic behavior of the Maxwell field $\Phi=\mu-\rho / r+\ldots$, where $\rho$ is charge density. It has been found that increase of $\mu$ at $T=0$ results in the insulator to metal transition, which in the gravity theory is related to Hawking-Page transition [23] between black soliton and black hole. Also superconductors with symmetries other than $s$-wave have been studied [24].

\subsection{Dark matter and $A d S / C F T$}

Of special interest are the papers studying the effect of dark matter field on such properties of superconductors as transition temperature, the condensate amplitude, upper critical field, coherence length, penetration depth, etc. One assumes that besides the Maxwell field there exists another U(1)-gauge field, which represents that part of the matter in the Universe, which is not visible with telescopes. The minimal gravitational action in $n$-dimensional space-time reads 


$$
S_{g}=\int \sqrt{-g} \mathrm{~d}^{n} x \frac{1}{2 \kappa^{2}}(R-2 \Lambda),
$$

where $\kappa^{2}=8 \pi G_{n}$ is an $n$-dimensional gravitational constant. The cosmological constant is given by $\Lambda=$ $-\frac{(n-1)(n-2)}{2 L^{2}}$, where $L$ is the radius of the AdS spacetime. The matter action contains the Abelian-Higgs sector coupled to the second $U(1)$-gauge field. It is provided by the following action:

$$
\begin{gathered}
S_{m}=\int \sqrt{-g} \mathrm{~d}^{n} x\left(-\frac{1}{4} F_{\mu \nu} F^{\mu \nu}-\left[\nabla_{\mu} \psi-\mathrm{i} q A_{\mu} \psi\right]^{\dagger}\right. \\
\left.\left[\nabla^{\mu} \psi-\mathrm{i} q A^{\mu} \psi\right]-V(\psi)-\frac{1}{4} B_{\mu \nu} B^{\mu \nu}-\frac{\alpha}{4} F_{\mu \nu} B^{\mu \nu}\right) .
\end{gathered}
$$

The scalar field potential representing the superconductor satisfies $V(\psi)=m^{2}|\psi|^{2}+\frac{\lambda_{\psi}}{4}|\psi|^{4} . \quad F_{\mu \nu}=2\left[\nabla_{\mu} A_{\nu}\right]$ stands for the ordinary Maxwell field strength tensor, while the second U(1)-gauge field $B_{\mu \nu}$ is given by $B_{\mu \nu}=$ $2 \nabla_{[\mu} B_{\nu]}$. Moreover, $m, \lambda_{\psi}, q$ represent mass, coupling constant, and charge related to the scalar field $\psi$, respectively. On the other hand, $\alpha$ is a coupling constant between U(1) fields. Analysis of the three-dimensional superconducting systems living in a $(3+1)$-dimensional space-time, requires $n=5$ dimensional gravity theory.

\subsection{Analogies and differences}

For condensed matter specialists it is important to note that the field $\Psi$ plays here a very similar role as (usually denoted by the same symbol) field in the GinzburgLandau approach. There is, however, a very important difference. While the condensation in the GinzburgLandau approach requires non-zero value of the quartic parameter $\lambda_{\psi}$, in the gravity theory the field $\Psi$ undergoes condensation for $\lambda_{\psi} \equiv 0$. The other crucial difference has to do with the mass parameter $m^{2}$. Its counterpart in the Ginzburg-Landau approach is usually denoted by $a$ and is assumed to change sign at the superconducting transition temperature: $a=a^{\prime}\left(T-T_{c}\right)$ taking on negative value in the superconducting state.

In a formal analogy, in the gravity approach $m^{2}<0$ in the superconducting state. However, the stability of the AdS $(n+1)$-dimensional space-time requires fulfilling the Breitenlohner-Freedman bound $m^{2}>-n^{2} / 4$. It means the lower limit on the the "mass" term in holographic superconductor. While it is generally accepted that the scalar field condenses [25] for $m^{2}<0$, there are papers proving the existence of the solution for $m^{2}>0$ in specific conditions. Kim et al. have shown [26] that the holographic superconductor may still exist for the case $m^{2}>0$. They found that if the mass of the scalar field increases, the system undergoes "the phase space or configuration space folding", i.e., the two very close boundary conditions can lead to the different states. For instance, one is with the broken symmetry while the other is a symmetric one.

The other tantalizing question is the inquire about the meaning of the limit for superconducting material, if any? Would its existence translate to the the limit of the super- conducting gap function? In the BCS approach the magnitude of the gap function is directly proportional to the electron-phonon coupling constant. However, the very large values of the coupling constant make the BCS weak coupling approach not valid. The Eliashberg method usually considered as a strong coupling theory, in fact takes retardation effects into account and is valid beyond BCS limit. However, based on the expansion with respect to electron-phonon coupling approach the theory is not a strong coupling one and possible instabilities at larger couplings have been analyzed and predicted. The detailed analysis of the relation between BreitenlohnerFreedman bound and the gaps in the field theory would shed additional light on the superconducting instability of materials, but this is outside the scope of the present paper.

\subsection{Some results}

Studying the influence of dark matter on the properties of superconductors we are mainly interested in the changes of their properties as characterized by the coupling constant $\alpha$ (see Eq. (2)). Working in the probe limit we have found that dark matter sector affects various properties of the holographic superconductors depending on their symmetry and other details. In particular the superconducting transition temperature $T_{c}$ of the $s$-wave superconductor with dark matter (DM) is modified as $T_{c}^{D M}=T_{c}+\tilde{\alpha} T_{0}$, where $T_{c}$ is the superconducting transition temperature without dark sector and $T_{0}$ is constant depending on the dimensionality of space-time and other model parameters, $\tilde{\alpha}=1-\alpha^{2} / 4$. Similar dependence for the model of SU(2) Yang-Mills $p$-wave holographic superconductor reads $T_{c}^{D M}=T_{c} / \tilde{\alpha}^{1 / 6}$, while in the Maxwell vector $p$-wave holographic superconductor with dark matter sector and for real components of the vector field gives us the same description of the phase transitions as the $s$-wave model [15-19]. It is worth noting the dependence of the superconducting transition temperature $T_{c}$ on the charge density $\rho$, which is $T_{c} \propto \rho^{1 / 3}$. The value $1 / 3$ of the power factor in a three-dimensional superconductor seem to be strong coupling modification of the exponent $2 / 3$ known from the Bose-Einstein condensation of charged local pair bosons in narrow band superconductors [27]. The coherence length $\xi$ and the penetration depth $\lambda$ both feature the dependence on the coupling $\alpha$ with $\lambda^{D M}=\lambda \sqrt{\tilde{\alpha}}$ and $\xi^{D M}=\xi / \sqrt{\tilde{\alpha}}$. As a result the presence of dark matter influences both length scales and the parameter $\kappa^{D M}=\tilde{\alpha} \kappa$. As $\kappa^{D M}$ may decrease with increasing $\alpha$ the superconductor may change [18] from the weakly second type (with $\kappa^{D M}>1 / \sqrt{2}$ ) to first kind $\left(\kappa^{D M}<1 / \sqrt{2}\right)$. If observed, this change ${ }^{\dagger}$ could be an important fingerprint of the effect of dark matter on real

${ }^{\dagger}$ We take the opportunity and correct the missprint in recent paper [18] which erroneously concludes that $\kappa$ is unaffected by the dark matter. 
superconductors and not only on the holographic ones. Most of the above results have been obtained in the probe limit, see however [28] for recent analysis of the effect of dark sector with back-reaction taken into account.

\section{Miscellaneous issues}

Very often we are asked about the motivation why to study the influence of a dark matter sector in holographic superconductors. Typically interlocutors admit that dark matter may play an important role in gravitational theories of the Universe and inquire: if it is important for superconductors; if and how the dark matter might be detected in the laboratory by studying real superconductors. Another important aspect of discussion is related to the fact that our Universe has the Einstein-de Sitter geometry, but hypothetical anti-de Sitter spacetime is assumed in the gauge/gravity correspondence. The above problems are at the heart of the gauge/gravity duality.

\subsection{Relation to real life systems}

The first question is whether the superconductors studied in the holographic theory have something to do with real life systems studied in the lab. We tend to believe that the AdS/CFT analogy describes or at least gives hints about the behavior of real systems. We have the following arguments in favor of this conviction:

(i) The duality has helped to understand the extremely low viscosity of the quark-gluon plasma. While perturbative approaches [29] fail to provide the understanding of the puzzle, the gauge-gravity duality finds the universal scale for the viscosity [30].

Applied to study superconductors the gauge-gravity duality describes the behavior expected to be observed in real life superconductors (phase transition, critical exponents, etc.). That it provides sensible and not random answers for various questions is an important argument in favor of its relevance to real world. We mention here but a few examples.

(ii) It shows the appearance of condensation at a given temperature with correct or close to correct temperature dependence of the parameter expected to describe the condensate [14] and the expected large value of the ratio between the gap and the superconducting transition temperature.

(iii) As a further example we quote very nontrivial predictions of the AdS/CFT theory which do agree with other (quantum critical) models [31]. In that work the calculations of the dynamical pair susceptibility by Zaanen and collaborators are presented. Moreover, the predictions are expected to be measured in the near future.

(iv) The analogy seem to provide deep understanding of the relations (scaling properties) observed near quantum critical points as observed in real materials. This is demonstrated e.g. in recent studies of incoherent transport in clean metals [32].
Summarizing this aspect, it seems that the AdS/CFT is saying something new about condensed matter problems in general and holographic superconductors in particular and this is encouraging. We accept the arguments of Anderson that many properties of superconductors [33] are "overdetermined by experimental facts" and that not every property has been adequately answered by the AdS/CFT duality. However, we think that the discussed applications provide an opportunity for field theories by finding solutions to strong coupling problems. At the same time the calculations provide real life applications of string theory ideas.

\section{2. $A d S / C F T$ vs. $d S$ universe}

The relation between the description of the system possessing conformal property by the field theory and by the gravity duality is really a tricky one and all the objections may eventually turn out well founded. Our understanding of the gauge-gravity duality is the following. Embedding the field theory into the AdS space-time with the ordinary matter enables us to calculate the properties of the system (which the field theory is supposed to describe) in the strong coupling [12] by perturbative methods of the gravity theory evaluated at the boundary. Thus we treat/consider the AdS/CFT correspondence as a kind of calculus, which enables relatively easy access to strong coupling limit of the CFT.

Using the AdS/CFT approach and studying the properties of holographic superconductors in the background with dark matter sector we rely on the following. First we accept that the gauge-gravity duality teaches us about strongly coupled superconductors which are produced and studied in the laboratories. Some examples of this can be found in [13]. The AdS/CFT correspondence relies on the AdS space time. Its validity to perform the calculations is not related whether the Universe in which the superconductors exist itself forms the AdS or de Sitter space-time. The dark matter existence seems to be obvious from astrophysical data as discussed in the Introduction. If it interacts with ordinary matter as proposed in Eq. (2) and quantified by the coupling constant $\alpha$, so it modifies the behavior of the ordinary matter. These modifications are found to influence the superconducting transition temperature and could, in principle, be observed as the annual changes of the properties of superconductors following the expected annual changes in the distribution of the dark matter [34].

\subsection{Dark matter}

Finally, we discuss the relation between the dark matter as required in astrophysics and its effect on the behavior of superconductors. The very notion of dark matter is an old one. The term first appeared in the paper by Koteyev and is used ever since [35]. Our understanding of the problem relies on the following arguments, supported by the observations and related work aimed at the detection of dark matter. There exist numerous astronomical observations which are described in terms of dark matter. 
The precise nature of it is not well known. As already mentioned, different particles have been proposed as the candidates. If the interpretation of astronomical observations in terms of dark matter is true, so dark matter must exist and, through its coupling - here $\alpha$ - will modify the properties of the ordinary matter and thus also the properties of superconductors. We try to pinpoint, how it may change the properties of the superconductors. If the calculated changes of the holographic superconductors are indeed expected to apply to real materials then there is a chance to observe these changes and thus get an information on the local dark matter distribution.

The most important question of the experimental Colleagues is related to the feasibility of the detection of the dark matter in the laboratory. We all accept the fact that the dark matter exists over there in Cosmos. But if the dark matter exists, as required by the astrophysical observations, so it is present not only in the macrocosm, but also in our neighborhood and may be spotted during the annual motion of the Earth [34]. The possible influence of the dark matter on superconductors can in principle be detected by precise and cleverly designed experiments looking at the annual changes of their properties. We rely here on the arguments presented in [34], where the authors analyze the annual modulations of the dark matter. Our additional assumption is that dark matter is non-homogeneously distributed in the neighborhood of the Sun [36] and these inhomogeneities will make a difference.

The gravity is a classical field theory. It means that there are no particles in it. The electromagnetism is described by the Maxwell theory. In the same manner we describe the dark matter as another classic field, analogous to the electromagnetic one. The same model we are using here as described in the previous section, has been recently studied in the context of particle physics [37]. These authors hope to observe the signatures of dark matter in the "planned polarized electron scattering experiments". The hope is that polarized electrons in those experiments will respond differently because of the dark matter. In the same mood, we do hope that electrons in superconductors will also "feel" the dark sector. Another question is related to the possible condensation of the dark matter itself. Indeed, the model with both matter and dark matter vortices has been recently studied [38], but the discussion of the results is outside the scope of the present paper.

\section{Conclusions}

We have discussed the general approach recently developed to study strongly coupled superconductors by means of the theory of gravity. The main goal of our study is to find the influence of dark matter on the properties of superconductors. In the approach the signatures of dark matter are visible as $\alpha$ dependence of various properties of superconductors. In the probe limit, when one neglects the back-reaction of the condensing field on the metric, the coupling strength $\alpha$ enters various parameters in a multiplicative way making it difficult to identify the presence of dark matter.

Combined with the evidence of dark matter in the inner Milky Way, its non-homogeneous distribution around the Sun provides a hope of dark matter detection in the precise experiments with superconductors. Similarly to the particle experiments aimed at the detection of dark matter using the fact that "The count rate should experience an annual modulation due to the relative motion of the Earth around the Sun" [34] we also expect that the properties of superconductor will show annual modulation. The application of the string theory to condensed matter problems is relatively recent endeavor. Despite that, many novel results have been obtained. However, much more has to be done. Let us finish with the following cautionary statements. The field of holographic superconductivity is a developing one and not all relevant questions have been asked and adequately answered. Similarly, the concept of dark matter requires a lot of theoretical studies and observations before it will be properly and completely understood. Our work [15-19] is directed towards better understanding of both: strongly coupled superconductors and seemingly omnipresent, but still illusory dark matter.

\section{Acknowledgments}

MR was partially supported by the grant of the National Science Center DEC-2013/09/B/ST2/03455 and KIW by the grant DEC-2014/13/B/ST3/04451.

\section{References}

[1] M. Tinkham, Introduction to Superconductivity, McGraw-Hill, New York 2004.

[2] P. Dirac, Proc. R. Soc. Lond. A 133, 60 (1931).

[3] B. Cabrera, Phys. Rev. Lett. 48, 1378 (1982).

[4] S. Burdin, M. Fairbairn, P. Mermod, D. Milstead, J. Pinfold, T. Sloan, W. Taylor, Phys. Rep. 582, 1 (2015).

[5] S. Courteau, M. Cappellari, R.S. de Jong, A.A. Dutton, E. Emsellem, H. Hoekstra, L.V.E. Koopmans, G.A. Mamon, C. Maraston, T. Treu, L.M. Widrow, Rev. Mod. Phys. 86, 47 (2014).

[6] P.A.R. Ade et al. (BICEP2/Keck and Planck Collaborations), Phys. Rev. Lett. 114, 101301 (2015).

[7] D. Hooper, S. Profumo, Phys. Rep. 453, 29 (2007).

[8] Ch. Beck, Phys. Rev. Lett. 111, 231801 (2013).

[9] F. Wilczek, New J. Phys. 16, 082003 (2014).

[10] G. Bertone, D. Hooper, J. Silk, Phys. Rep. 405, 279 (2005).

[11] J.M. Maldacena, Int. J. Theor. Phys. (1999).

38, 1113

[12] S. Sachdev, Ann. Rev. Condens. Matter Phys. 3, 9 (2012).

[13] A.G. Green, Contemp. Phys. 54, 33 (2013). 
[14] S.A. Hartnoll, C.P. Herzog, G.T. Horowitz, Phys. Rev. Lett. 101, 031601 (2008).

[15] Ł. Nakonieczny, M. Rogatko, Phys. Rev. D 90, 106004 (2014).

[16] Ł. Nakonieczny, M. Rogatko, K.I. Wysokiński, Phys. Rev. D 91, 046007 (2015).

[17] Ł. Nakonieczny, M. Rogatko, K.I. Wysokiński, Phys. Rev. D 92, 066008 (2015).

[18] M. Rogatko, K.I. Wysokiński, J. High Energy Phys. 12, 041 (2015)

[19] M. Rogatko, K.I. Wysokiński, arXiv:1508.02869.

[20] R.A. Klemm, A. Luther, M.R. Beasley, Phys. Rev. B 12, 877 (1975).

[21] R. Gregory, S. Kanno, J. Soda, J. High Energy Phys. 10, 010 (2009); L. Barclay, R. Gregory, S. Kanno, P. Sutcliffe, J. High Energy Phys. 12, 029 (2010); S. Kanno, Class. Quantum Grav. 28, 127001 (2011).

[22] J. Jing, Q. Pan, S. Chen, J. High Energy Phys. 11, 045 (2011); J. Jing, Q. Pan, B. Wang, Phys. Rev. D 84, 126020 (2011); J. Jing, B. Wang, Q. Pan, S. Chen, ibid. 83, 066010 (2011)

[23] T. Nishioka, S. Ryu, T. Takayanagi, J. High Energy Phys. 03, 131 (2010).

[24] R.G. Cai, L. Li, L.F. Li, R.Q. Yang, Sci. ChinaPhys. Mech. Astron. 58, 060401 (2015).

[25] Y. Brihaye, B. Hartmann, Phys. Rev. D 83, 126008 (2011).
[26] Y. Kim, Y. Ko, S-J. Sin, Phys. Rev. D 80, 126017 (2009).

[27] R. Micnas, J. Ranninger, S. Robaszkiewicz, Rev. Mod. Phys. 62, 113 (1990).

[28] Yan Peng, Qiyuan Pan, Yunqi Liu, arXiv:1512.08950.

[29] E. Shuryak, Prog. Part. Nucl. Phys. 53, 273 (2004).

[30] P.K. Kovtun, D.T. Son, A.O. Starinets, Phys. Rev. Lett. 94, 111601 (2005).

[31] J.-H. She, B.J. Overbosch, Y.-W. Sun, Y. Liu, K.E. Schalm, J.A. Mydosh, J. Zaanen, Phys. Rev. B 84, 144527 (2011).

[32] R.A. Davison, B. Gouteraux, S.A. Hartnoll, J. High Energy Phys. 10, 112 (2015).

[33] P.W. Anderson, Phys. Today 66, 9 (2013).

[34] K. Freese, M. Lisanti, Ch. Savage, Rev. Mod. Phys. 85, 1561 (2013).

[35] C.S. Frenk, S.D.M. White, Ann. Phys. (Berlin) 524, 507 (2012).

[36] G. Jungman, M. Kamionkowski, K. Griest, Phys. Rep. 267, 195 (1996).

[37] H. Davoudiasl, H.-S. Lee, W.J. Marciano, Phys. Rev. D 85, 115019 (2012).

[38] P. Ariasa, F.A. Schaposnik, J. High Energy Phys. 12, 011 (2014) 